\title{
Identifying isochrones in GPR profiles from DEP-based forward modeling
}

\author{
Olaf EISEN, Frank WILHELMS, Uwe NIXDORF, Heinrigh MILLER \\ Alfred-Wegener-Institut für Polar- und Meeresforschung, Postfach 120161, D-27515 Bremerhaven, Germany \\ E-mail: oeisen@awi-bremerhaven.de
}

\begin{abstract}
Isochronic continuous horizons between 20 and $90 \mathrm{~m}$ depth in a groundpenetrating radar (GPR) profile, recorded in Dronning Maud Land, Antarctica, are identified by comparison of synthetic and measured single radar traces. The measured radargram is derived from a stacked GPR profile; the synthetic radargram is computed by convolution of the complex reflection coefficient profile, based on dielectric profiling (DEP) data of a $150 \mathrm{~m}$ ice core, with a depth-invariant wavelet. It reproduces prominent reflections of the measured radargram to a considerable degree. Analyzing matching peaks in both radargrams enables us to identify isochronic reflections and transfer individual volcanic-event datings to the GPR profile. Reflections are primarily caused by changes in permittivity; changes in conductivity are of minor importance. However, several peaks in permittivity and conductivity show a good correlation and indicate that some reflections are related to acidic layers. The results demonstrate the possibility of reproducing radargrams from ice-core property profiles, a necessary step for the interpretation of remotely sensed radar data and the general significance of connecting ice-core and radar data for correct interpretations. Problems related to forward modeling, data gaps, origin of permittivity peaks, and GPR profiles used for comparison, are discussed.
\end{abstract}

\section{INTRODUGTION}

The European Project for Ice Coring in Antarctica (EPICA) aims at retrieving two deep ice cores in different regions of the Antarctic ice sheet. Drilling has been performed at Dome Concordia since 1996; the second deep drilling operation started in 2001 at the new Kohnen station in Dronning Maud Land (DML), near the site DML05. Variations in the spatial distribution of precipitation and ice-sheet dynamics make it necessary to use additional information for accurate interpretation of ice-core data, and their extension to neighboring regions. Spatial accumulation rates can be obtained from snow pits, shallow ice cores and space-borne remote sensing (Karlöf and others, 2000; Sommer and others, 2000; Wahr and others, 2000; Zwally and others, 2001). The interpretation of the internal ice-sheet structure in terms of accumulation rates can be accomplished by ground-penetrating radar (GPR) surveys (Richardson and others, 1997; Nereson and others, 2000; Siegert and Hodgkins, 2000; Kanagaratnam and others, 2001).

Electromagnetic (EM) waves penetrating the ice are partially reflected at boundaries where the complex dielectric constant changes, mainly due to variations in ice density and chemical composition. Assuming that a continuous internal reflection horizon (IRH) corresponds to an isochronous layer, the spatial variation of layer depth provides information on variations in the accumulation rate and changes due to ice-sheet dynamics.

Dating of IRHs is achieved by converting GPR profiles from travel time to depth domain, using EM velocity-depth relations (Jezek and Roeloffs, 1983; Clarke and Bentley, 1994; Richardson and others, 1997; Hempel and others, 2000;
Eisen and others, 2002), and transferring age-depth relations, usually obtained from snow pits or ice cores (Oerter and others, 1999; Sommer and others, 2000), to prominent IRHs. Other studies have demonstrated the direct connection between volcanic events and IRHs (e.g. Millar, 1981; Bogorodsky and others, 1985; Siegert, 1999; Hempel and others, 2000), but a direct comparison between synthetic radargrams based on ice-core data and measured radargrams is still pending (Moore, 1988; Miners and others, 1997).

In this study, we demonstrate the possibility of reproducing prominent IRHs in a GPR radargram by forward modeling, identify most as isochrones, some of which are related to acidic signals, like volcanic eruptions, and transfer the dating to continuous horizons in a GPR profile. The data were obtained during several EPICA pre-site surveys in DML. In 1998/99, numerous GPR profiles were recorded, connecting the locations of ice cores drilled in earlier seasons. High-resolution dielectric profiling (DEP) along the ice core B32, retrieved at DML05 in 1997/98 (Oerter and others, 2000), forms the basis for calculating synthetic radargrams with a general frequency domain convolution using a depth-invariant wavelet.

\section{GPR SURVEY ANALYSIS}

Common-offset GPR measurements were performed with a commercial $200 \mathrm{MHz}$ monopulse bistatic RAMAC system (Malå Geoscience, Sweden). The antennae were mounted on a sled and pulled by a Skidoo at an average speed of $8 \mathrm{~km} \mathrm{~h}^{-1}$, passing the borehole location B32 in a distance of a few decimeters; a distance meter trigged the transmitter 


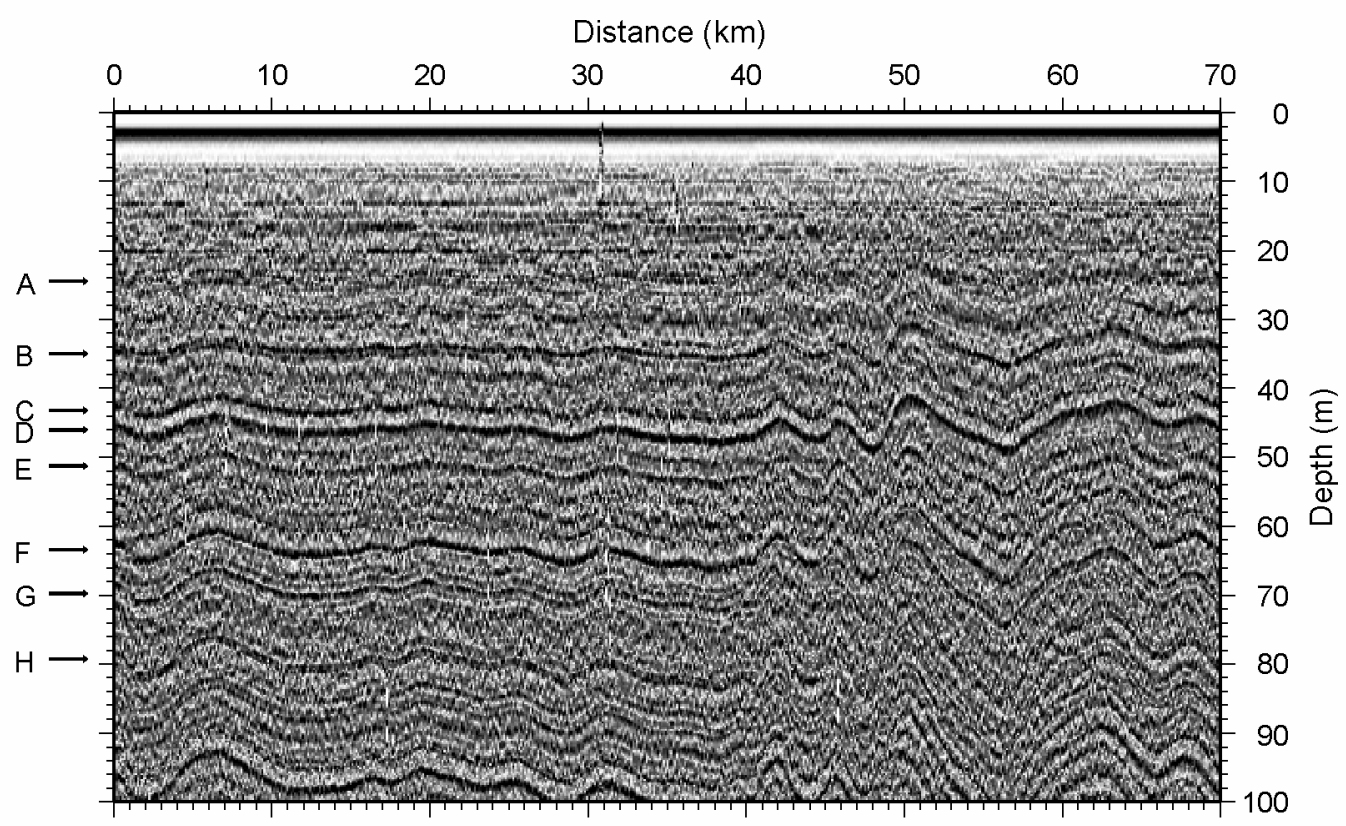

Fig. 1. Example of a filtered, stacked and gain-corrected GPR profile, recorded with unshielded $200 \mathrm{MHz}$ antennae. The profile runs from the drill site B32 at DML05 to DML15; traces were recorded every meter. Dominant horizons are labeled $A-H$ and indicated by arrows; depth is in respect to the 1998 surface. The vertical line in the upper part near $k m 32$ is caused by a hardware failure of the system.

pulse at an interval of $1 \mathrm{~m}$. Each trace consists of eight vertically stacked pulse recordings of a 1500 ns two-way traveltime (TWT) window containing 2400 samples.

Tracking of coherent patterns in adjacent traces makes it possible to identify prominent reflectors in processed GPR profiles (Fig. 1). Processing includes five-fold horizontal stacking, band-pass filtering, and automatic gain control (AGG). Conversion from TWT to depth domain is achieved by applying a velocity-depth distribution derived from common-midpoint (CMP) measurements (Eisen and others, 2002). In the resulting profile, numerous continuous prominent IRHs can be identified below $20 \mathrm{~m}$ depth, each $1.5-3 \mathrm{~m}$ wide. They will be referred to later when comparing GPR and synthetic radargrams.

Due to the relatively low signal-to-noise ratio (SNR) and lateral inhomogeneity of the firn pack, it is difficult to locate the arrival time of IRHs exactly when considering a single GPR trace for comparison with a synthetic radargram. To increase the SNR of the GPR data for further analysis, and to minimize the influence of reflections from obstacles at the surface (e.g. a weather station and metal stakes), we create a single trace radargram, $S_{\mathrm{GPR}}$, used for later comparison, by stacking all traces from 11 different GPR profiles located within a radius of $50 \mathrm{~m}$ of B32. Before stacking, the traces are shifted to the primary signal of the direct airwave, resampled at a sample rate of $0.5 \mathrm{~ns}$. After stacking and dewowing, a $100 \mathrm{~ns}$ AGC filter is applied to the trace to correct for device-related direct-current components, geometric spreading, absorption, etc.

\section{FORWARD MODELING OF RADARGRAMS}

The forward modeling of impulse measurements generally considers the distribution of reflection coefficients with depth to be the impulse response function of the subsurface. The convolution of the transmitted signal with the impulse response function results in the recorded trace, as described below.

\section{Ice-core DEP data}

The complex relative dielectrical constant can be written as

$$
\varepsilon=\varepsilon^{\prime}-i \varepsilon^{\prime \prime}=\varepsilon^{\prime}-i \frac{\sigma}{\varepsilon_{0} \omega}=|\varepsilon| \mathrm{e}^{-i \delta},
$$

where the real part $\epsilon^{\prime}$ is the ordinary relative permittivity of the medium. The imaginary part $\varepsilon^{\prime \prime}$ is the dielectric loss factor and can be expressed as a function of conductivity $\sigma$, angular frequency $\omega$, and the permittivity of vacuum $\varepsilon_{0}$. The last expression defines the loss tangent, $\tan \delta=\varepsilon^{\prime \prime} / \varepsilon^{\prime}$.

Along an ice core, $\varepsilon$ can be determined by means of DEP (Moore and Paren, 1987). An improved DEP device developed by Wilhelms and others (1998), and further refined by Wilhelms (2000), essentially a calibrated guarded scanning capacitor, allows the simultaneous measurement of both components of $\varepsilon$. This device was used at a frequency of $250 \mathrm{kHz}$ to determine $\varepsilon$ along the ice core B32 in $\Delta z=$ $5 \mathrm{~mm}$ increments with a systematic accuracy of about $1 \%$ and a statistical error of $\sim 0.1 \%$ for each component, and an accuracy of $1 \mathrm{~cm}$ in depth (Fig. 2). The depth error results from the positioning of the $1 \mathrm{~m}$ long core sections in the measuring bench.

Several schemes to reject sections with poor core quality, and thus false DEP data, were investigated. The best compromise between least rejection and least disturbed convolution signals is obtained by calculating running mean and standard deviation within a $2.5 \mathrm{~m}$ window along the core. DEP values that show a permittivity which is more than one standard deviation below the window mean are rejected, as the lower permittivity values are most likely caused by cracks in the ice.

Following this procedure, about $3.5 \%$ of the DEP data with an average section length of $2 \mathrm{~cm}$ had to be removed in the upper $100 \mathrm{~m}$ of the core. In total, $4.5 \%$ of the DEP data are missing. 


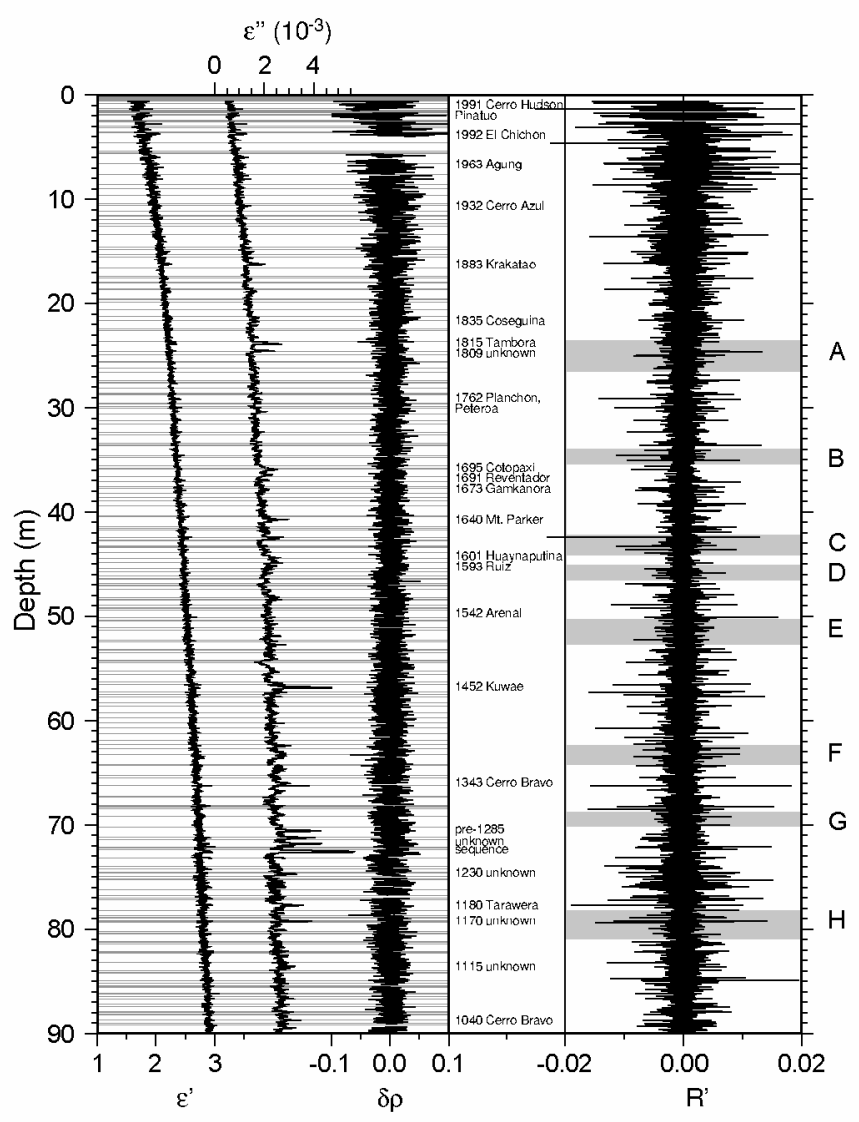

Fig. 2. Depth distribution of the measured ordinary relative permittivity $\varepsilon^{\prime}$; dielectric loss factor $\varepsilon^{\prime \prime}$, scaled to $200 \mathrm{MHz}$; density variation $\delta \rho$; the real part $R^{\prime}$ of the complex reflection coefficient; and name and date of identified volcanic events of the ice core B32. The density variation is calculated from $\gamma$ absorption measurements, corrected for core breaks, in respect to the boxcar filtered mean density of a $5 \mathrm{~m}$ window. The wide grey horizontal bars labeled $A-H$ on the right indicate the depth and vertical extent of the most prominent reflection horizons in the GPR profile (Fig. 1) near B32, corrected to the 1998 surface. The narrow grey horizontal bars indicate gaps in the DEP data record.

\section{The complex reflection coefficient}

The complex reflection coefficient at an interface of two media with different dielectrical properties is determined by their complex impedance contrast. The reflection coefficient of two adjacent layers with complex dielectric constants $\varepsilon_{k}$ and $\varepsilon_{k+1}$ measured at depths $k \Delta z$ and $(k+1) \Delta z$, respectively, is given by

$$
R_{k+\frac{1}{2}}=R_{k+\frac{1}{2}}^{\prime}+i R_{k+\frac{1}{2}}^{\prime \prime}=\frac{\sqrt{\varepsilon_{k}}-\sqrt{\varepsilon_{k+1}}}{\sqrt{\varepsilon_{k}}+\sqrt{\varepsilon_{k+1}}},
$$

which can be separated and rearranged to obtain $R_{k+\frac{1}{2}}^{\prime}$ and $R_{k+\frac{1}{2}}^{\prime \prime}$ as a function of $\left|\varepsilon_{k}\right|,\left|\varepsilon_{k+1}\right|, \delta_{k}$ and $\delta_{k+1}$. The index $k+\frac{1}{2}$ means that the corresponding depth value for $R$ is the mean depth of both data points, i.e. $\left(k+\frac{1}{2}\right) \Delta z$.

Several tests show that gaps larger than several $\Delta z$ produce too low reflection coefficients when using a linear interpolation, and too large reflection coefficients when applying different spline interpolation schemes. To avoid artificial values, the DEP data are linearly interpolated in gaps that are $\sim 3 \Delta z$ in length or smaller, and $R$ is calculated from Equation (2). If the gap length exceeds this limit, $R$ is set to 0 (Fig. 2). We consider this to be a more accurate way of treating missing DEP data than to interpolate $\varepsilon$ on an equidistant grid before calculating the reflection coefficient, as the change in $\varepsilon$ is more important than the actual value. Support for this procedure comes from test runs with downsampled DEP data, as, with a new sample interval of $\sim 5 \Delta z$, significant changes in the reflection characteristics are already evident.

Analogous to the TWTof a reflected radar pulse in ice, a propagation time for each data point of the $R(\Delta z)$ series can be calculated from the in situ EM wave speed, $c=c_{0} / \sqrt{\varepsilon^{\prime}}$, with $c_{0}$ being the wave speed in vacuum. The resulting non-equidistant series is then projected onto an equidistant grid by means of a linear interpolation and a time increment $\Delta t=\Delta z / c_{\text {ice }}=29.7 \mathrm{ps}$, yielding the series $R(\Delta t)$ ( $\Delta t$ is the time necessary for an EM wave to propagate the DEP sampling distance of $5 \mathrm{~mm}$ in ice with $c_{\text {ice }}=$ $1.68 \times 10^{8} \mathrm{~ms}^{-1}$ (Bogorodsky and others, 1985)).

\section{Radar wavelets}

Numerous authors emphasize the crucial role of the transmitted radar wavelet for forward modeling of radargrams and GPR processing (Moore, 1988; Arcone and others, 1995; Hildebrand, 1996). We investigate several wavelets from raw GPR data, among which are the direct airwave, reflections from the ice-shelf-water boundary, and several internal reflections. Although the EM reflections from the ice-shelf-sea-water boundary represent a close image of the original reflected pulse, this wavelet only partly reproduces reflections in the upper $30 \mathrm{~m}$ of the ice using DEP data. The most likely reason for this is the different physical in situ properties in the upper layers of the ice shelf in comparison to site DML05 on the polar plateau. The wavelet was recorded near Neumayer station, with refrozen meltwater at the surface and solid ice underneath. Thus, absorption, dispersion, phase shifts, etc. lead to a different wavelet shape, and thus to different reflections. Best results are obtained with a wavelet determined from a strong internal reflection recorded during a CMP survey near DML05, which is resampled using an Akima spline interpolation at 2048 equidistant points and a time increment $\Delta t$ as above (Fig. 3). This results in the wavelet series $W(\Delta t)$ we use for further calculations.

\section{Frequency domain convolution}

The convolution of the wavelet series $W(\Delta t)$ with the reflection coefficient series $R(\Delta t), S_{\mathrm{DEP}}=W * R$, is carried out in the frequency domain by multiplying their fast Fourier transforms. The resulting synthetic DEP radargram $S_{\mathrm{DEP}}(\Delta t)$ is transformed to depth by applying the inverse TWT-depth conversion introduced above.

\section{RESULTS}

Isochrones are layers of equal age that obtained a similar characteristic at the surface on a regional scale, which is sustained during vertical advection and deformation. The accurate dating of GPR profiles requires the identification of isochronic IRHs, which can best be achieved by identifying matching peaks in the measured GPR and synthetic DEP radargrams. If the peaks are related to chemical origin, the corresponding IRHs are isochrones, and, in the case of volcanic events, the dating can be transferred to the GPR profile. As firn age is monotonically increasing with depth, adjacent layers which are parallel to isochronic IRHs have 

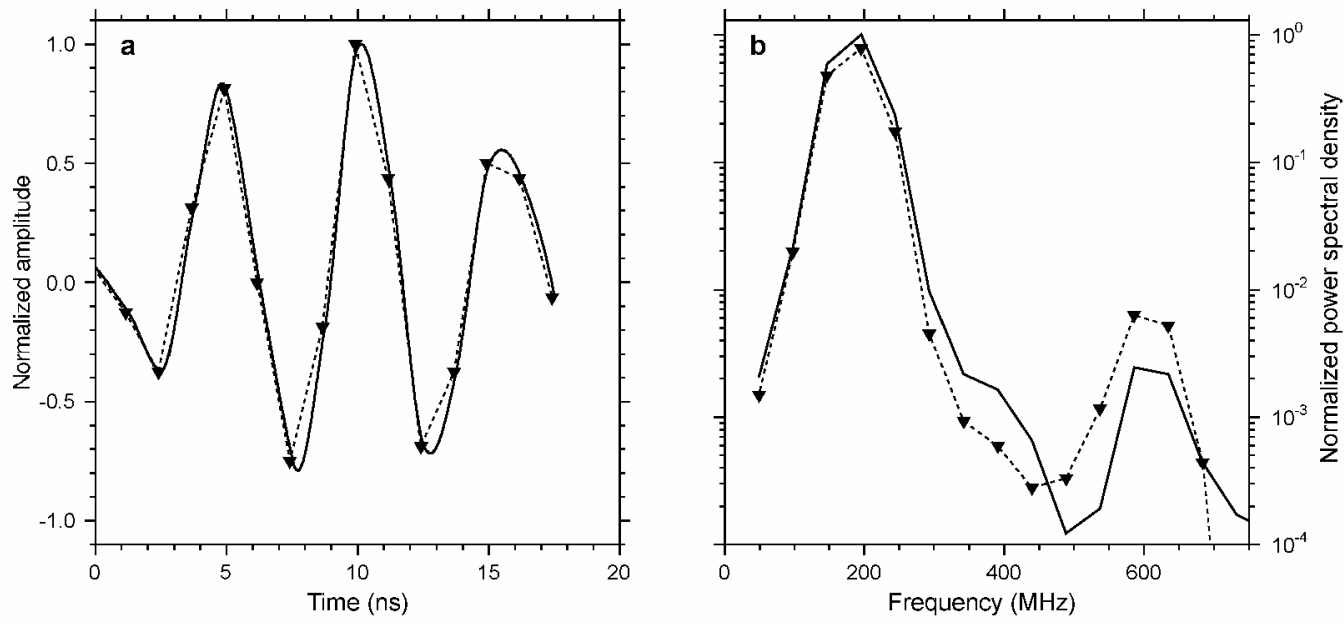

Fig. 3. Time-domain signals ( $a$ ) and power spectrum ( $b$ ) of the raw-data wavelet (thin dashed line with triangles) determined from an internal reflection, and the interpolated resampled wavelet used for the convolution (thick line).

to be isochrones as well. In the following we will carry out this procedure for the prominent IRHs selected in Figure 1.

\section{DEP vs GPR radargram}

For the direct comparison of both radargrams we use their squared envelopes, which are proportional to energy, calcuated from the single trace radargrams. Due to the influence of the direct air and ground wave as well as antenna ringing, signals with a TWT $<50 \mathrm{~ns}(\sim 10 \mathrm{~m})$ are neglected in the following comparison.

Most prominent IRHs in the GPR profile (events A-H, Fig. 1) are visible in $S_{\mathrm{GPR}}$ as strong peaks (Fig. 4). Some, however, have rather small amplitudes (e.g. event E), but can nevertheless be clearly identified as continuous signals in the GPR profile.

In general, the power envelope of the synthetic trace $S_{\text {DEP }}$ shows a good agreement in numerous incidents with the power envelope of $S_{\mathrm{GPR}}$, enabling the identification of distinct reflections. However, some outliers are also present on either side. Matching partners of comparable size to the GPR envelope are visible in the DEP envelope for events A, B, C, F and G. A very good agreement, even in phase structure, exists for events A, Fand G. Events B and E have $S_{\mathrm{DEP}}$ envelope peaks of only about half the width of the $S_{\mathrm{GPR}}$ signal, and their maxima are shifted by 0.5 and $-1.0 \mathrm{~m}$, respectively. For events $\mathrm{C}$ and $\mathrm{F}$, the directly matching envelope peak is of the same size, but while event $\mathrm{C}$ is preceded by a stronger signal which is not visible in $S_{\mathrm{GPR}}$, event $\mathrm{F}$ is followed by one. A direct partner in $S_{\mathrm{DEP}}$ is missing for event $\mathrm{D}$, and the peak in $S_{\mathrm{DEP}}$ is smaller in size and with a slightly shifted maximum for event $\mathrm{H}$, but still comparable in phase. The most obvious outliers in $S_{\mathrm{DEP}}$ occur at 16.5, 49 and $64 \mathrm{~m}$. We now discuss probable reasons for these differences.

\section{Qualitative error analysis}

Numerous factors affect the shape of the synthetic and the recorded radargrams. The most obvious problems are missing DEP data, strongly altering interference patterns of reflected wave trains, and the presence of noise in the GPR profiles.

Comparison studies with single GPR traces or small ensembles of stacked traces were only partly successful. The GPR processing sequence described above makes use of coherent positions of reflecting horizons with depth with- in a radius of $50 \mathrm{~m}$ around $\mathrm{B} 32$, i.e. time shifts during the recording due to surface of reflector roughness are smaller than half a wavelength $\left(0.5 \mathrm{~m}\right.$ for $\left.c=2.0 \times 10^{8} \mathrm{~ms}^{-1}\right)$.

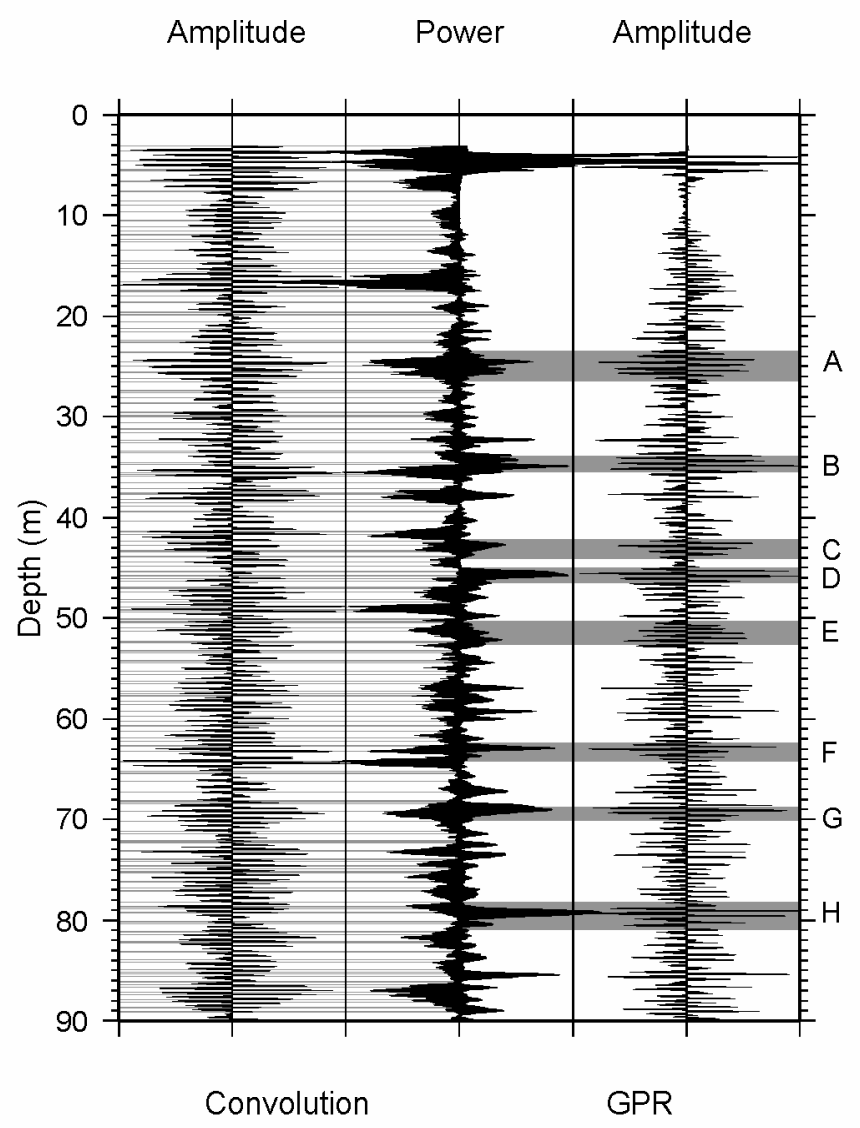

Fig. 4. Variable-amplitude wiggle plot of the synthetic DEP radargram $S_{\mathrm{DEP}}$ (left) and the measured gain-corrected GPR radargram $S_{\mathrm{GPR}}$ (right). In the middle, the squared envelopes of each radargram are shown, with the power of $S_{\mathrm{DEP}}$ increasing to the left and $S_{\mathrm{GPR}}$ increasing to the right. The GPR signal is corrected to the 1998 surface; the first $3 \mathrm{~m}$ have been muted because of the influence of the direct and ground wave. A $10 \mathrm{~cm}$ wide mean boxcar filter has been applied to the envelopes to increase clarity. Wide grey bars in the right half labeled $A-H$ indicate prominent reflection horizons in the GPR profile (Fig. 1); narrow grey bars in the left half indicate gaps in the DEP data (Fig. 2). 
Although stacking of more than 500 traces significantly increases the SNR and emphasizes dominant horizons, weaker signals might be destroyed.

The original DEP data, on the other hand, represent a point measurement, which therefore has a low SNR but a high vertical resolution. Lateral inhomogeneities are present, especially in the upper part of the firn, where sastrugi are still distinguishable and might lead to a reflectivity over one Fresnel zone different than that calculated from the DEP data. Moreover, parts of the data are missing, and cannot be interpolated easily without introducing artificial reflection properties. Gaps of a few centimeters in length might already result in the loss of considerable information on reflectivity. For example, four gaps with a total length of $8 \mathrm{~cm}$ occur within event D. Three of these gaps occur at points where $\varepsilon$ seems to change significantly, suggesting that the strong peak of $S_{\mathrm{GPR}}$ at $45.5 \mathrm{~m}$ lacks a matching peak in $S_{\text {DEP }}$ because of wrong values for $R$. Likewise, event $\mathrm{H}$ is interrupted in the DEP data by five gaps.

Additional causes for differences of both radargrams come along with the convolution scheme. In general, the propagating wave changes shape due to dispersion effects, and a complex reflection coefficient causes phase shifts at each layer boundary. The use of a constant wavelet neglects both processes. Sensitivity runs with different wavelets indicate that the reflection characteristics for longer travel times depend on the wavelet choice (e.g. with a different signal the single DEP reflection at $86 \mathrm{~m}$ is strongly reduced in magnitude and becomes a multi-peak signal). Moreover, interferences due to multiple reflections are not accounted for by a simple convolution. This could be the reason for the sharp peaks in $S_{\text {DEP }}$ at 49 and $64 \mathrm{~m}$, which do not have any peak in $S_{\mathrm{GPR}}$. The broad peak between 16.0 and $17.5 \mathrm{~m}$, on the other hand, is matched by two much smaller and sharper GPR peaks, and might be the result of constructive interference caused by missing reflectivity and multiple reflections, or negative interference in the GPR because of pronounced lateral inhomogeneities at this depth.

The AGC applied to the GPR trace basically compensates for energy losses due to geometric spreading. As pointed out by Hildebrand (1996), absorption and reflection losses as well as focusing are of minor importance in the upper part of the considered depth range, and are therefore not major factors for discrepancies. At larger travel times, however, the reflected GPR signal is close to the noise level, unavoidably decreasing the SNR, and thus resulting in larger differences in magnitude of matching peaks.

\section{Physical origin of reflections}

To reveal the physical origin of the observed matching synthetic reflections, we perform two sensitivity studies. For the calculation of the synthetic radargram $S_{\mathrm{DEP}}^{\mathrm{I}}$ of the first study, $\varepsilon^{\prime \prime}$ is smoothed with a $20 \mathrm{~m}$ boxcar mean filter and $\varepsilon^{\prime}$ is left unchanged. For the second study, resulting in $S_{\mathrm{DEP}}^{\mathrm{II}}$, selected individual peaks in $\varepsilon^{\prime}$, that show a correlation with conductivity signals at same depths, are smoothed and $\varepsilon^{\prime \prime}$ is left unchanged.

The synthetic radargram $S_{\mathrm{DEP}}^{\mathrm{I}}$ is quasi-identical to the original $S_{\mathrm{DEP}}$. The second study demonstrates that amplitudes of reflections in $S_{\mathrm{DEP}}^{\mathrm{II}}$ are 1-2 orders of magnitude smaller than prominent peaks in $S_{\mathrm{DEP}}$ at the same depth. Moreover, because of the increased variability of the conductivity record, the positions of reflections in $S_{\mathrm{DEP}}^{\mathrm{II}}$ show a less clear agreement with $S_{\mathrm{GPR}}$, i.e. they cannot explain the observed matching IRHs of $S_{\mathrm{DEP}}$ and $S_{\mathrm{GPR}}$. These results confirm earlier findings (Moore, 1988; Hildebrand, 1996; Miners and others, 1997) that the reflection coefficient is dominated by changes in permittivity and that conductivity changes are negligible. As we relate the origin of reflections to changes in permittivity coinciding with acidic layers, two questions arise: what is the cause of the correlation between chemical impurities and permittivity? and do acidic layers affect the permittivity of the firn?

We rule out measurement artifacts related to the relaxation frequency of ice, as the DEP processing scheme has been extensively tested in this respect (Wilhelms, 2000). In some cases (e.g. the Coseguina $(1835 ; 21.7 \mathrm{~m}$ ) (Nicaragua) and Tambora (1815; $23.8 \mathrm{~m}$ ) (Indonesia) events), the $\gamma$-absorption density record shows distinct peaks in density as well. In the case of the adjacent unknown eruption (1809; $24.5 \mathrm{~m}$ ), a comparable peak in density is missing (Fig. 2). The DEP-based density at the same depth, calculated with the complex mixing model (Wilhelms, 2000), i.e. corrected for dielectrical mixing of density and conductivity of the complex $\varepsilon$ of firn, shows the same pattern. This indicates that the correlations between acidic peaks in conductivity, permittivity and density are not systematic, but that different mechanisms are present, as pointed out by Fujita and others (2000).

The simplest explicative process is of meteorological origin (e.g. accumulation coming along with unusual circulation patterns, increasing chemical impurities and changing snow properties simultaneously). Nevertheless, complex dielectrical mixing between the air and snow phase, changes in the firn lattice, or protonic defects, related to chemical impurities, might play a role at different frequencies, requiring further investigations on the microphysical level.

\section{Identifying isochrones}

The ice core B32 has been dated by counting annual layers in various chemical records (Sommer and others, 2000), and volcanic events have been identified by Göktaş (2002) by a combination of annual-layer counting, nss-sulphate concentrations and identified $\mathrm{H}_{2} \mathrm{SO}_{4}$ depositions (Fig. 2).

Having related the physical origin of matching peaks of $S_{\mathrm{GPR}}$ and $S_{\mathrm{DEP}}$ to permittivity peaks in the $\varepsilon$-depth distributions (Fig. 2) enables us to connect certain chemical events with dominant signals in the DEP radargram. Because of the comparable structure of several permittivity and conductivity peaks, we have to assume that these permittivity peaks are related to volcanic eruptions or other chemical events, with the consequence that the corresponding IRHs are isochrones. The ice-core dating can then be transferred via several matching peaks to the GPR radargram and further to the GPR profile. It has to be kept in mind that the strongest peak of an IRH observed in the radargram is slightly shifted to larger travel times, or depths, as the wavelet maximum is delayed from the first arrival by $\sim 10 \mathrm{~ns}$, corresponding to about $2 \mathrm{~m}$.

Of the set of prominent IRHs we selected for our analysis, the double peak of event $\mathrm{A}$ is coincident with the Tambora and one unknown eruption in 1815 and 1809, respectively. Events $\mathrm{C}$ and $\mathrm{F}$ coincide with strong peaks in $\varepsilon^{\prime \prime}$, which result from above-normal values in several chemical species (dated to 1620 and 1375, respectively), but which make it difficult to attribute these signals solely to volcanic eruptions (personal communication from H. Oerter, 2002). 
Although for event $\mathrm{H}$ the matching peak is of less good quality than at shallower depths, it is striking that two eruptions, Tarawera, New Zealand, in 1180 and one unknown in 1170, are dominant at the same depth. Event $\mathrm{D}$ is coincident with the Ruiz (Colombia) eruption (1593), but as a corresponding peak in $S_{\mathrm{DEP}}$ is missing, the origin of the IRH is unclear.

To summarize, six out of the eight strongest IRHs evident from the GPR profile show matching peaks in GPR- and DEP-based radargrams, all of which are caused by peaks in permittivity. Four of these coincide with signals in the conductivity of chemical origin, with a very good correlation to permittivity, two of which are attributed to volcanic events. This evidence strongly suggests that these four events are isochrones. As all dominant observed continuous IRHs in the considered depth range are parallel to adjacent identified isochrones, they must be of isochronous origin as well.

\section{GONGLUSION}

Based on a simple convolution scheme, we are able to calculate a synthetic radargram from high-resolution DEP icecore data, which reproduces dominant features of a measured radargram to a considerable degree. In four cases, the ice-core dating from single chemical events can be directly transferred to continuous IRHs in common-offset GPR profiles, via matching dominant peaks in the radargrams. The dated horizons provide an independent means of synchronizing ice cores from different locations, and can be used to determine the regional and temporal distribution of the accumulation rates.

All IRHs are caused by changes in the permittivity. However, major IRHs in the depth range 20-90 $\mathrm{m}$ are associated with volcanic eruptions or distinct chemical events of other origin, that seem to cause changes in the chemical as well as physical properties.

Discrepancies between the synthetic and real radargram are associated with gaps in the DEP data, the presence of noise in the GPR data, and lack of consideration of important physical phenomenon during wave propagation by the convolution scheme. To overcome data gaps, detailed studies on the random structure of the DEP profiles on a regional scale, and their influence on EM reflections are required to develop interpolation procedures that successfully reproduce missing data without introducing artificial EM reflections.

Instead of developing more sophisticated convolution schemes, we suggest using finite-difference forward modeling for calculating synthetic radargrams, as the simulation of the propagation and reflection processes of the EM waves (e.g. multiple reflections, phase shifts and absorption) are implicitly accounted for. Currently, work is in progress to calculate finite-difference-based radargrams from the DEP data used for this study.

\section{ACKNOWLEDGEMENTS}

We acknowledge the important contribution of the field parties during data acquisition. The data would not have been acquired without the continuing maintenance of the radar system by G. Stoof. Preparation of this work was supported by the Deutsche Forschungsgemeinschaft grant Ni493/1 and two scholarships of the Studienstiftung des Deutschen Volkes. The final manuscript profited from the valuable comments of J. C. Moore. The contribution of N. Azuma (Scientific Editor) and one anonymous reviewer is gratefully acknowledged. This work is a contribution to the "European Project for Ice Coring in Antarctica" (EPICA), a joint European Science Foundation/European Commission (EC) scientific programme, funded by the $\mathrm{EC}$ and by national contributions from Belgium, Denmark, France, Germany, Italy, the Netherlands, Norway, Sweden, Switzerland and the United Kingdom. This is EPICA publication no. 54.

\section{REFERENCES}

Arcone, S. A., D. E. Lawson and A. J. Delaney. 1995. Short-pulse radar wavelet recovery and resolution of dielectric contrasts within englacial and basal ice of Matanuska Glacier, Alaska, U.S.A. 7. Glaciol., 41(137), 68-86.

Bogorodsky, V.V., C. R. Bentley and P. E. Gudmandsen. 1985. Radioglaciology. Dordrecht, etc., D. Reidel Publishing Co.

Clarke, T. S. and C. R. Bentley. 1994. High-resolution radar on Ice Stream B2, Antarctica: measurements of electromagnetic wave speed in firn and strain history from buried crevasses. Ann. Glaciol., 20, 153-159.

Eisen, O., U. Nixdorf, F. Wilhelms and H. Miller. 2002. Electromagnetic wave speed in polar ice: validation of the common-midpoint technique with high-resolution dielectric-profiling and $\gamma$-density measurements. Ann. Glaciol., 34, 150-156.

Fujita, S., T. Matsuoka, T. Ishida, K. Matsuoka and S. Mae. 2000. A summary of the complex dielectric permittivity of ice in the megahertz range and its applications for radar sounding of polar ice sheets. In Hondoh, T., ed. Physics of ice core records. Sapporo, Hokkaido University Press, 185-212.

Göktaş, F. 2002. Characterisation of glacio-chemical and glacio-meteorological parameters of Amundsenisen, Dronning Maud Land, Antarctica. Ber. Polarforsch./Rep. Pol. Res. 425.

Hempel, L., F. Thyssen, N. Gundestrup, H.B. Clausen and H. Miller. 2000. A comparison of radio-echo sounding data and electrical conductivity of the GRIP ice core. F. Glaciol., 46(154), 369-374.

Hildebrand, A. 1996. Untersuchung der Laufzeit- und Amplitudenverhalten elektromagnetischer Impulse bei glaziologischen Radarmessungen. (Ph.D. thesis, Westfälische Wilhelmsuniversität Münster.)

Jezek, K. C. and E. A. Roeloffs. 1983. Measurements of radar wave speeds in polar glaciers using a down-hole radar target technique. Cold Reg. Sci. Technol., 8(2), 199-208.

Kanagaratnam, P., S. P. Gogineni, N. Gundestrup and L. Larsen. 2001. High-resolution radar mapping of internal layers at the North Greenland Ice Core Project. 7. Geophys. Res., 106(D24), 33,799-33,811.

Karlöf, L. and 13 others. 2000. A 1500 year record of accumulation at Amundsenisen, western Dronning Maud Land, Antarctica, derived from electrical and radioactive measurements on a $120 \mathrm{~m}$ ice core. F. Geophys. Res., 105(D10), 12,471-12,483.

Millar, D. H. M. 1981. Radio-echolayering in polar ice sheets and past volcanic activity. Nature, 292(5822), 441-443.

Miners, W. D., A. Hildebrand, S. Gerland, N. Blindow, D. Steinhage and E.W. Wolff. 1997. Forward modeling of the internal layers in radio echo sounding using electrical and density measurements from ice cores. $\mathcal{F}$. Phys. Chem., Ser. B, 101(32), 6201-6204.

Moore, J. C. 1988. Dielectric variability of a $130 \mathrm{~m}$ Antarctic ice core: implications for radar sounding. Ann. Glaciol., 11, 95-99.

Moore, J. C. and J. G. Paren. 1987. A new technique for dielectric logging of Antarctic ice cores. F. Phys. (Paris), 48, Colloq. C1, 155-160. (Supplément au 3.)

Nereson, N. A., C. F. Raymond, R. W. Jacobel and E. D. Waddington. 2000. The accumulation pattern across Siple Dome, West Antarctica, inferred from radar-detected internal layers. f. Glaciol., 46(152), 75-87.

Oerter, H., W. Graf, F. Wilhelms, A. Minikin and H. Miller. 1999. Accumulation studies on Amundsenisen, Dronning Maud Land, by means of tritium, dielectric profiling and stable-isotope measurements: first results from the 1995-96 and 1996-97 field seasons. Ann. Glaciol., 29, 1-9.

Oerter, H. and 6 others. 2000. Accumulation rates in Dronning Maud Land, Antarctica, as revealed by dielectric-profiling measurements of shallow firn cores. Ann. Glaciol., 30, 27-34.

Richardson, C., E. Aarholt, S.-E. Hamran, P. Holmlund and E. Isaksson. 1997. Spatial distribution of snow in western Dronning Maud Land, East Antarctica, mapped by a ground-based snow radar. 7. Geophys. Res., 102(B9), 20,343-20,353

Siegert, M.J. 1999. On the origin, nature and uses of Antarctic ice-sheet radio-echo layering. Prog. Phys. Geogr., 23(2), 159-179.

Siegert, M.J. and R. Hodgkins. 2000. A stratigraphic link across $1100 \mathrm{~km}$ of the Antarctic ice sheet between the Vostok ice-core site and Titan Dome (near South Pole). Geophys. Res. Lett., 27(14), 2133-2136. 
Sommer, S. and 9 others. 2000. Glacio-chemicalstudy spanning the past $2 \mathrm{kyr}$ on three ice cores from Dronning Maud Land, Antarctica. 1. Annually resolved accumulation rates. 7. Geophys. Res., 105(D24), 29,411-29,421.

Wahr, J., D. Wingham and C. Bentley. 2000. A method of combining ICESat and GRACE satellite data to constrain Antarctic mass balance. $\mathcal{F}$. Geophys. Res., 105(B7), 16,279-16,294.
Wilhelms, F. 2000. Messung dielektrischer Eigenschaften polarer Eiskerne. Ber. Polarforsch./Rep. Pol. Res. 367, 1-171.

Wilhelms, F., J. Kipfstuhl, H. Miller, K. Heinloth and J. Firestone. 1998. Precise dielectric profiling of ice cores: a new device with improved guarding and its theory. F. Glaciol., 44(146), 171-174.

Zwally, H.J. and 15 others. 2001. ICESat's laser measurements of polar ice, atmosphere, ocean, and land. F. Geodyn., 34(3-4), 405-445. 